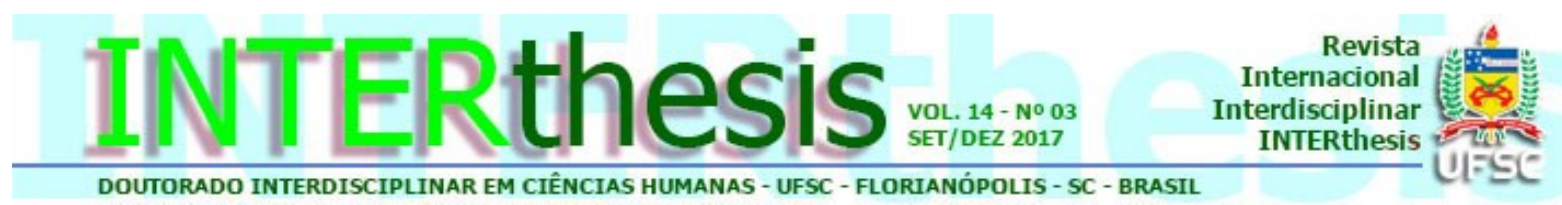

\title{
ETNOCIÊNCIA DA CIÊNCIA: A BUSCA POR SIMETRIA NA PESQUISA CIENTÍFICA
}

\section{Resumo:}

Raphael Jonas Cypriano ${ }^{1}$ Reinaldo Duque Brasil Landulfo Teixeira ${ }^{2}$

A ciência tem por objetivo estudar todos os fenômenos de nosso planeta, auxiliandonos na compreensão da realidade. Do ponto de vista institucional, a ciência é uma organização coletiva constituída por objetos de pesquisa e sujeitos pesquisadores que exibem um sistema de crenças (kosmos), saberes (corpus) e práticas (práxis) fortemente influenciado pelos conhecimentos produzidos dentro da própria comunidade científica. Essa conjuntura humana é pouco compreendida, apesar de exercer forte influência na visão de mundo transmitida pela ciência para toda a sociedade. Nesse contexto, o presente artigo defende a necessidade de se fazer uma 'Etnociência da Ciência', a qual garanta a possibilidade da comunidade científica ser estudada cientificamente.

Palavras-chave: Método Científico. Domínio Cognitivo. Epistemologia. Simetria. Estudo da Natureza.

\section{CIÊNCIA: UMA CONSTRUÇÃO HUMANA DA REALIDADE}

A ciência moderna surgiu na Europa, nos séculos XVI e XVII, emergindo da efervescência provocada pela Renascença na organização econômica, política e social da época. As raízes dessa ciência têm como alguns de seus grandes ícones René Descartes, Francis Bacon e Isaac Newton. O primeiro destacou-se por sua ideia racionalista e metodológica para se conhecer a realidade; o segundo, pela elaboração de um método experimental objetivo para pesquisar os fenômenos naturais; e o último, pela capacidade de unir, na prática, estas duas ideias num método racionalista e experimentalista aplicável para estudar o mundo natural (KUHN, 1998; MORAIS, 2007).

Essa matriz epistêmica conduziu àquilo que hoje conhecemos como o 'mito da razão absoluta'. Este se refere à crença na infinita capacidade racional do ser humano, além de realçar a experimentação como único meio válido de descobrir a

\footnotetext{
${ }^{1}$ Mestre em Ecologia pela Universidade Federal de Viçosa. Assessor Técnico da ONG Articulação no Semiárido (ASA), Viçosa, MG, Brasil E-mail: raphael.cypriano@yahoo.com.br

2 Doutor em Botânica pela Universidade Federal de Viçosa. Professor Adjunto do Instituto de Ciências da Vida, Universidade Federal de Juiz de Fora, Governador Valadares, MG, Brasil E-mail: rduquebrasil@yahoo.com.br
} 
ordem e o funcionamento da natureza, sendo os resultados desta via metodológica expressos por meio de quantidades e relações matemáticas. Neste contexto, os fatos observados se tornam independentes do observador, construindo um mundo essencialmente objetivo (CAPRA, 2006). A ciência, ao longo do tempo, integrou-se progressivamente à técnica e à tecnologia, introduzindo-se no âmago das sociedades. E nesse processo, o papel da ciência adentrou em uma dimensão de ação inimaginável na realidade humana (MORIN, 2008).

Entretanto, quando estudamos profundamente a ciência como uma construção social, isto é, uma atividade humana destinada à produção de conhecimentos, algumas perguntas surgem: O que é o conhecimento científico? Quem o produz e como ele é construído?

Segundo o biólogo Maturana (2001), o uso de explicações científicas pelos membros de uma comunidade para validar suas afirmações define a ciência como um domínio cognitivo. Dessa maneira, o meio científico não é diferente de outras esferas cognitivas, pois é caracterizado por um campo de ações, em que um critério de validação ou aceitabilidade é utilizado, pelos membros da sociedade, para aceitar suas hipóteses como 'verdadeiras'.

Dentro dessa conjuntura, é importante ressaltar a forma como a sociedade científica constrói e valida seu conhecimento. De acordo com Maturana (2001), explicamos um fenômeno pela ciência formal no momento em que se satisfazem quatro condições:

1. Ter o fenômeno a explicar ou uma pergunta a responder ( $\mathrm{O}$ quê?): este surge no emocional do cientista, que é um sujeito interessado em descobrir e explicar o 'novo'.

2. Elaborar uma hipótese explicativa para este fenômeno (Por quê?): esta é construída se baseando em conhecimentos científicos já creditados como verdadeiros, ou socialmente aceitos.

3. Utilizar uma metodologia para testar a hipótese (Como?): esta se fundamenta em trabalhos já realizados, utilizando métodos considerados científicos, geralmente seguindo a lógica do 'quanto mais objetivo melhor'.

4. Analisar os resultados obtidos e sintetizar conclusões: o experimento originará dados quantitativos a serem interpretados estatisticamente. A comparação destes resultados com os de outros trabalhos, já realizados na

R. Inter. Interdisc. INTERthesis, Florianópolis, v.14, n.3, p. 01-13 Set.-Dez. 2017 
área, irá informar sobre sua 'veracidade' e 'legitimidade'. Assim, a hipótese de trabalho será refutada ou não e a comunidade acadêmica terá uma resposta respaldada cientificamente.

Esses são os principais pilares que sustentam o kosmos (sistema de crenças e cosmovisões), corpus (sistemas de conhecimentos sistematizados) e praxis (sistema de práticas e comportamentos) da comunidade científica. Nessa organização, os cientistas vão aperfeiçoando sua experiência da mesma forma que outras comunidades ditas tradicionais: com "o que Ihe disseram" (experiência historicamente acumulada), com "o que lhe dizem" (experiência socialmente compartilhada) e com o que observa por si mesmo do fenômeno natural que estudam (experiência individual) (TOLEDO; BARRERA-BASSOLS, 2010).

Mas será que ao final das etapas de uma pesquisa científica fica 'comprovado' que um fenômeno existe, ou será que apenas temos evidências de como um fenômeno pode ocorrer sob determinada situação experimental e sob determinada visão de mundo? Será que a ciência consegue explicar todos os fenômenos existentes no planeta através de seu arcabouço teórico e metodológico? E este conhecimento dito científico seria 'melhor', mais 'verdadeiro', mais próximo da 'realidade' quando comparado a outros sistemas de kosmos, corpus e praxis?

A objetividade pode ser considerada uma forma adequada de análise, já que é determinada por observações repetidas e verificações concordantes. Nesse sentido, o conhecimento científico é correto somente na medida em que se baseia em dados verificados e está apto a fornecer previsões de acontecimentos dentro de condições controladas. Contudo não deixa de ser uma das maneiras que o ser humano encontrou de construir seu próprio universo - simbólico - que lhe permite compreender e interpretar o mundo à sua volta e interferir a seu favor na realidade que o cerca, criando ferramentas para melhorar seu modo de viver e de interagir com a natureza (CASSIRER, 1972).

Rubem Alves (2007) destaca que a ciência é o que os cientistas fazem, ou seja, é a atividade de construção de conhecimentos exercida por um grupo social bem definido: os cientistas. Sendo assim, metaforicamente, o conhecimento científico pode ser considerado tudo aquilo que pode ser pescado no "rio da realidade" com uma rede chamada método científico. No entanto, o autor realça que toda rede, independentemente da seletividade e eficiência de sua malha, deixa R. Inter. Interdisc. INTERthesis, Florianópolis, v.14, n.3, p. 01-13 Set.-Dez. 2017 
passar mais coisas do que pode pegar. Portanto, os resultados da pesquisa científica geralmente restringem-se apenas ao que fica retido pelas redes do método, ignorando os fenômenos que não podem ser controlados e desconsiderando o que não foi pescado e não pode ser explicado com as ferramentas utilizadas.

Segundo Heisenberg (1980), um dos maiores físicos do séc. XX, parece ser pretensão da ciência intervir eficazmente no universo com um método que isole e ilumine cada fenômeno, avançando de uma para outra relação de fatos. O progresso das certezas científicas não caminha na direção de uma grande certeza. A física do séc. $X X$ demonstrou que todos os conceitos e teorias são aproximados e limitados, além disso, confirmou que o sujeito é uma das variáveis que pode interferir nos fenômenos atômicos, colocando em cheque as ideias estritamente objetivas da realidade defendidas pelo cartesianismo.

Nesse contexto, a ciência atual tem necessidade não apenas de um pensamento apto a considerar a complexidade do real, mas desse mesmo pensamento para considerar sua própria complexidade e a complexidade das questões que ela levanta para a humanidade (MORIN, 2008).

\section{EM BUSCA DA SIMETRIA EPISTÊMICA}

A ciência é uma construção humana e uma poderosa instituição social que influencia diretamente a vida de todos nós. Contudo não buscamos estudar e compreender mais a fundo esta organização de forma crítica, sendo as afirmações científicas aceitas como verdades absolutas pela maior parte da população sem grandes questionamentos. Isto é evidenciado pelo poder atual da expressão: 'comprovado cientificamente', que exibe uma grande influência no pensamento e na conduta das pessoas.

Quando analisamos mais profundamente o kosmos, corpus e práxis da comunidade científica, nos deparamos com o fato de que são os próprios cientistas que fazem suas ciências, seus discursos sobre a ciência, sua ética da ciência e suas políticas da ciência. No entanto, a conjuntura e a estrutura da própria comunidade científica são muito pouco estudadas e questionadas. Ou seja, o conhecimento científico é um conhecimento que não se conhece (etno) cientificamente. Essa R. Inter. Interdisc. INTERthesis, Florianópolis, v.14, n.3, p. 01-13 Set.-Dez. 2017 
constituição humana que desenvolveu metodologias tão surpreendentes e hábeis para apreender todos os objetos a ele externos, não dispõe de métodos para se conhecer e pensar sobre si mesmo (LATOUR; WOOLGAR, 1997; MORIN, 2008).

A abertura de espaços na ciência para uma relação dialética entre a pesquisa e os pesquisadores, ou seja, entre objetos e sujeitos que estudam este objeto podem complementar o conhecimento científico. Nessa perspectiva, a análise das condições sociais, dos contextos culturais, dos sistemas de crenças e dos modelos de organização da investigação científica ocupa papel relevante na pesquisa. O estudo da estrutura da comunidade científica pode revelar aspectos da produção de conhecimentos que são influenciados por tendências de pensamentos e comportamentos proliferadas no âmago da própria ciência (MORAIS, 2007; SOUSA SANTOS, 2002).

Nessa linha de pesquisa, destaca-se a ideia de simetria do antropólogo Bruno Latour (1994). Segundo ele, vivemos atualmente numa sociedade que acredita ser 'moderna', caracterizada pela assimetria criada por duas grandes divisões arbitrárias: a primeira entre natureza e cultura, consequentemente entre ciências naturais e ciências humanas/sociais, e a segunda entre 'nós' ('modernos') e 'eles' ('primitivos'). Apenas nós, sociedade com uma visão eurocêntrica, etnocêntrica e cartesiana do mundo, diferenciamos de forma absoluta natureza e cultura, ciência e sociedade, enquanto que em grande parte das outras culturas esses elementos são quase coextensivos. A sociedade também é construída, tanto quanto a natureza, e é preciso compreender como as duas são, ao mesmo tempo, imanentes e transcendentes.

A busca pela simetria inicia-se quando estudamos simultaneamente a produção da natureza e dos humanos que estudam esta natureza, ocupando uma posição intermediária. O objetivo do princípio da simetria não é apenas o de estabelecer a igualdade, ou seja, o de regular a balança no ponto zero (entre ciência e sociedade científica), mas também o de gravar as diferenças, as assimetrias que surgem quando contexto e conteúdo científico são confrontados (LATOUR, 1994).

Essas ideias compactuam com as de Einstein (1936), um dos cientistas mais admirados do mundo. Segundo ele, a relação recíproca entre a epistemologia e a ciência é de uma espécie notável, em que uma depende da outra. A epistemologia sem contato com a ciência torna-se um esquema vazio, bem como a ciência sem 
epistemologia é primitiva e confusa. De acordo com Einstein, quando procuramos um fundamento mais sólido para um fenômeno, devemos tentar tornar claro para si próprio de forma crítica os conceitos que utilizamos e as práticas que realizamos.

Os trabalhos de Latour e Woolgar (1997), Sá (2005) e Cypriano et al. (2013) apontam a importância de se estudar a comunidade científica e suas técnicas sobre um ponto de vista crítico, chocando a pesquisa científica com as práticas e pensamentos dos próprios cientistas. Nesse processo fica evidente a atuação de interesses, ambições, desejos, preconceitos, conservadorismos, crenças, fantasias, tendências e aspirações dos cientistas influenciando o direcionamento e as prioridades das pesquisas e, logo, a produção de conhecimentos. Latour e Woolgar (1997) realizaram uma longa pesquisa etnográfica dentro de um laboratório de neuroendocrinologia utilizado como modelo conceitual e espaço de observação social, e demonstraram como a sociedade científica é organizada, quais técnicas ela emprega para validar os fenômenos da natureza e qual a linguagem de 'signos' utilizada nos textos científicos.

\section{INTERESSES E PRECONCEITOS CIENTÍFICOS}

Quando refletimos de forma crítica sobre a estrutura científica, a ciência começa a revelar-se como uma entidade que direciona suas energias para estudar alguns fenômenos em detrimento de outros. Assim sendo, alguns aspectos da realidade são mais bem estudados somente pelo fato dos próprios cientistas quererem isso. Essa característica aflora com mais clareza quando tratamos de fenômenos que abordem de alguma forma aspectos que confrontam a visão de mundo científica e/ou que necessitam de novos conceitos e práticas para serem compreendidos, como o efeito da música em plantas abordado por Cypriano et al. (2013).

Algumas áreas de conhecimento enfrentam mais esse conservadorismo e, logo, possuem grande dificuldade de ter seu conhecimento estudado e validado cientificamente, sendo negligenciadas pelos cientistas. Como exemplos, temos: homeopatia, acupuntura, floral, fitoterapia, efeito de técnicas de imposição de mãos (Reiki, passes e Johrei) e influências astrológicas em organismos vivos, como defendido pela Agricultura Biodinâmica. Essas áreas de conhecimento apresentam

R. Inter. Interdisc. INTERthesis, Florianópolis, v.14, n.3, p. 01-13 Set.-Dez. 2017 
seus próprios sistemas de kosmos, corpus e práxis e quando tentam 'comprovar cientificamente' seus conhecimentos, são desafiadas nas etapas de construção de uma pesquisa científica.

O método científico normalmente é o fator central de dificuldade, uma vez que esses sistemas citados podem não ser contemplados pelos métodos hoje existentes e respaldados pela comunidade científica. Dessa forma, são obrigados a criar novos métodos de avaliação de seu conhecimento e estes terão que ser aceitos pelos cientistas. A homeopatia enfrenta esse problema em suas pesquisas científicas quando utiliza métodos da farmacologia clássica, reducionista, mecanicista e materialista, focadas no princípio-ativo das plantas e curvas dose-resposta (VAN WASSENHOVEN, 2007). Outro exemplo é o estudo do efeito dos ritmos lunares no crescimento de plantas que também apresentam desafios na elaboração de um método científico (JOVCHELEVICH; CÂMARA, 2008).

Além disso, há dificuldades em construir hipóteses e discussões baseadas em conhecimentos científicos já estabelecidos, uma vez que essas outras áreas têm como base conhecimentos novos e pioneiros para o meio científico, com uma matriz de conceitos e ideias que não são ou são pouco abordados em trabalhos científicos. Por isso, uma parte dos cientistas acredita que essas linhas de pesquisas ditas como esotéricas ou metafísicas não são passíveis de acurácia científica e, portanto, são negligenciadas.

Novas ideias precisam de um campo intelectual aberto:

\begin{abstract}
Se é verdade que o surgimento e o desenvolvimento de uma nova ideia precisam de um campo intelectual aberto, onde se debatam e se combatam teorias e visões do mundo, se é verdade que toda novidade se manifesta como desvio [...] então o desenvolvimento científico, no sentido de que esse termo comporte necessariamente invenção e descoberta, necessita fundamentalmente de duas condições: 1) manutenção e desenvolvimento do pluralismo teórico (ideológico, filosófico) em todas as instituições e comissões científicas; 2) proteção do desvio, ou seja, tolerar/favorecer os desvios no seio dos programas e instituições (MORIN, 2008, p.35).
\end{abstract}

Segundo o físico Thomas Kuhn (1998), a novidade no meio científico somente emerge com muita dificuldade, que se manifesta através de resistências. Muitos conhecimentos hoje aceitos dentro da academia foram depreciados e até perseguidos durante seu surgimento, incluindo a Teoria da Evolução de Charles Darwin. A acupuntura, uma técnica oriental de cura baseada num conceito energético do corpo humano que tem como ponto principal o sistema de Chackras, é 
um exemplo de área de conhecimento que sofreu perseguição por parte da comunidade científica dos médicos brasileiros, quando esse método foi introduzido no país. Contudo, no momento em que os benefícios dessa técnica começaram a ser validados cientificamente, os médicos do Brasil tentaram restringir sua aplicação à classe médica (ROCHA et al., 2015). Isso mostra como a sociedade científica, ao mesmo tempo, que deprecia outros sistemas de kosmos, corpus e práxis, pode se apropriar de suas práticas e conhecimentos quando estes são validados cientificamente e são de seu interesse.

\section{POR UMA ETNOCIÊNCIA DA CIÊNCIA}

Todos os exemplos citados acima mostram a complexidade do meio social científico e a necessidade de compreendermos melhor e mais profundamente sua estrutura. No contexto de integração entre estudos naturais e sociais, surgem as Etnociências a partir da década de 50. As Etnociências podem ser consideradas ramos das ciências que nasceram do entrecruzamento entre a Sociolingüística, a Antropologia Cognitiva e as Ciências Naturais, e lidam com o estudo dos sistemas culturais (MARQUES, 2001). Neste sentido, segundo Cardona (1985, p.10), é uma área de pesquisa transdisciplinar que estuda "[...] todas as formas de classificação que o homem escolheu para dar ordem e nome àquilo que ele vê em torno de si $[\ldots] "$.

A etnociência é um campo de diálogo entre sujeitos com diferentes kosmos, corpus e práxis, porém historicamente sempre concentraram seus estudos em etnias indígenas e outras comunidades consideradas 'tradicionais', ou povos ditos 'primitivos' a partir de uma ótica 'moderna' (ALVES; SOUTO, 2010). Contudo, segundo Latour (2000), o prefixo 'etno' transpõe este contexto, abrigando diferentes abordagens na interação do pesquisador com o 'outro', aspecto chave dos estudos etnocientíficos. Uma de suas aplicações envolve essa transformação do pesquisador (observador) em pesquisado (observado), em que os cientistas são estudados por seus pares acadêmicos. Nessa nova relação, a alteridade tende a ser menor entre os sujeitos (cientista-cientista) em diálogo quando comparada ao contato entre um cientista e um xamã, por exemplo. 
Nessa perspectiva, este trabalho vem ressaltar a necessidade de expansão de estudos dentro da linha da Etnociência da Ciência que visa complementar o "conhecimento das coisas" com o "conhecimento do conhecimento das coisas", como destacado por Sousa Santos (2002). Investigando práticas, condutas, pensamentos, crenças e interesses dos cientistas sobre sua área de pesquisa e sobre seus objetos de pesquisa pode-se melhor compreender a essência da pesquisa científica enquanto atividade humana. Essa proposta tem por finalidade abrir um novo caminho, em que a esfera dos objetos e dos sujeitos que estudam estes objetos é investigada simultaneamente.

Nosso tempo exige um posicionamento que busque equilíbrio entre a crítica a os elogios, avaliando a ciência em sua condição de produto humano e enxergando ao mesmo tempo sua grandeza e sua problematicidade (MORAIS, 2007; SOUSA SANTOS, 2002). Importante destacar que alguns dos maiores críticos da ciência não são os sociólogos, antropólogos ou epistemólogos, são sim os próprios cientistas que estão em seus laboratórios ou em campo enxergando as 'assimetrias' que surgem na produção do conhecimento científico. Dentre estes cientistas, podemos destacar muitos renomados, como Albert Einstein (Nobel de Física, 1922), Werner Heisenberg (Nobel de Física, 1932), llya Prigogine (Nobel de Química, 1977), além de Humberto Maturana, Thomas Kuhn e Fritjop Capra.

Quando estudamos mais seriamente a comunidade científica, confrontando sujeitos e objetos, contradições e assimetrias emergem, fazendo-nos compreender mais profundamente a essência da ciência e da visão de mundo defendida por ela. Espera-se que, a partir das reflexões propostas neste artigo sobre a ciência enquanto um sistema de kosmos, corpus e práxis, a comunidade acadêmica seja incentivada a se perguntar: O que estudar? Por que fazer ciência? Como lidar com conhecimentos ainda não estudados e compreendidos pelos domínios da ciência? Como respeitar e dialogar com outros sistemas de kosmos, corpus e práxis? Como fazer ciência comprometida com sua função social? E, finalmente, fazer ciência com quem e para quem? 


\title{
ETHNOSCIENCE OF SCIENCE: THE SEARCH FOR SYMMETRY IN SCIENTIFIC RESEARCH
}

\begin{abstract}
:
Science aims to study all phenomena of our planet, helping us to understand the reality. From an institutional point of view, science is a collective organization consisting of research objects and researchers who exhibit belief (kosmos), knowledge (corpus) and practice (praxis) systems strongly influenced by the knowledge produced within the scientific community. This juncture is poorly understood, despite having a strong influence on the worldview transmitted by science for the whole society. In this context, this article defends the need to develop an 'Ethnoscience of Science', which could ensure the possibility for the scientific community to be studied scientifically.

Keywords: Scientific Method. Cognitive Domain. Epistemology. Symmetry. Nature Study.
\end{abstract}

\section{ETNOCIENCIA DE LA CIENCIA: LA BÚSQUEDA POR SIMETRÍA EN LA INVESTIGACIÓN CIENTÍFICA}

\section{Resumen:}

La ciencia tiene como objetivo estudiar los fenómenos de nuestro planeta y nos ayuda a comprender la realidad. Desde el punto de vista institucional, la ciencia es una organización colectiva que consiste en objetos de investigación y sujetos investigadores que exhiben un sistema de creencias (kosmos), conocimientos (corpus) y prácticas (praxis) fuertemente influenciadas por el conocimiento producido dentro de la comunidad científica. Esta coyuntura humana es poco conocida, a pesar de tener una fuerte influencia en la visión del mundo transmitida por la ciencia para la sociedad. En este contexto, el presente artículo defiende la necesidad de hacer una 'Etnociencia de la Ciencia', que asegura la posibilidad de la comunidad científica ser estudiada científicamente.

Palabras clave: Método Científico. Dominio Cognitivo. Epistemología. Simetría. Estudio de la Naturaleza. 


\section{REFERÊNCIAS}

ALVES, Angelo Giuseppe Chaves; SOUTO, Francisco José Bezerra. Etnoecologia ou Etnoecologias? Encarando a diversidade conceitual. In: ALVES, Angelo Giuseppe Chaves; SOUTO, Francisco José Bezerra; PERONI, Nivaldo (Org.). Etnoecologia em perspectiva: natureza, cultura e conservação. Recife: NUPEEA, 2010. p. 1739.

ALVES, Rubem. Entre a ciência e a sapiência: o dilema da educação. 18. ed. São Paulo: Edições Loyola, 2007.

BRASIL. Ministério da Casa Civil. Subchefia para Assuntos Políticos. Política Nacional de Desenvolvimento Sustentável dos Povos e Comunidades Tradicionais. Decreto 6.040 de 7 de fevereiro de 2007.

CAPRA, Fritjop. O Ponto de Mutação: a ciência, a sociedade e a cultura emergente.São Paulo: Cultrix, 2006.

CARDONA, Giorgio Raimondo. La Foresta di Piume, Manuale de Etnoscienza. Roma: Laterza, 1985.

CASSIRER, Ernst. Antropologia Filosófica: Ensaio sobre o homem. 1. ed. São Paulo: MesteJou, 1972.

CYPRIANO, Raphael Jonas; DUQUE-BRASIL, Reinaldo; KUKI, Kacilda Naomi; GODOY, Alice Godoy; REZENDE, Maíra Queiroz. A música pode influenciar as plantas? Qual a opinião de professores universitários sobre essa interação?Polêm!ca, Rio de Janeiro, v. 12, n. 1, p. 54-63, 2013. Disponível em: $<$ http://www.e-publicacoes.ueri.br/index.php/polemica/article/view/5275/3868>. Acesso em: 16 mar. 2014.

EINSTEIN, Albert. Physics and reality. Journal of the Franklin Institute, U.S.A., v. 221, p. 349-382, 1936.

HEISENBERG, Werner Karl. A imagem da natureza na física moderna. Lisboa: Ed. Livros do Brasil, 1980.

JOVCHELEVICH, Pedro; CÂMARA, Francisco Luis Araújo. Influência dos ritmos lunares sobre o rendimento de cenoura (Daucuscarota), em cultivo

R. Inter. Interdisc. INTERthesis, Florianópolis, v.14, n.3, p. 01-13 Set.-Dez. 2017 
biodinâmico. Revista Brasileira de Agroecologia, Botucatu/SP, v. 3, n. 1, p. 49-57, 2008. Disponível em:

<http://orgprints.org/27592/1/Jovchelevich Influ\%C3\%AAncia.pdf $>$. Acesso em: 27 out. 2016.

KUHN, Thomas Samuel. A estrutura das revoluções científicas. 5 ed. São Paulo: Editora Perspectiva S.A, 1998.

LATOUR, Bruno. Jamais fomos modernos: ensaio de antropologia simétrica. Rio de Janeiro: Editora 34, 1994.

LATOUR, Bruno. Ciência em ação: como seguir cientistas e engenheiros sociedade afora.São Paulo: UNESP, 2000.

LATOUR, Bruno; WOOLGAR, Steve. A vida de laboratório: a produção dos fatos científicos. Rio de Janeiro: Relume Duramá, 1997.

MARQUES, José Geraldo. O Olhar (Des)Multiplicado: O Papel do Interdisciplinar e do Qualitativo na Pesquisa Etnobiológica e Etnoecológica. In: Anais do Seminário de Etnobiologia e Etnoecologia do Sudeste, 1. ed., Rio Claro: UNESP, p. 47-92, 2001.

MATURANA, Humberto. Cognição, ciência e vida cotidiana. Belo Horizonte: Ed. UFMG, 2001.

MORAIS, Régis de. Evoluções e revoluções da ciência atual.Campinas/SP: Editora Alínea, 2007.

MORIN, Edgar. Ciência com consciência. 11. ed. Rio de Janeiro: Bertrand Brasil, 2008.

ROCHA, Sabrina Pereira; DE BENEDETTO, Maria Auxiliadora Craice;

FERNANDEZ, Fabíola Holanda Barbosa; GALLIAN, Dante Marcelo Claramonte. A trajetória da introdução e regulamentação da acupuntura no Brasil: memórias de desafios e lutas. Ciência \& Saúde Coletiva, São Paulo, v. 20, n. 1, p. 155-164, 2015. Disponível em: <http://www.scielo.br/scielo.php?pid=S141381232015000100155\&script=sci abstract\&tlng=pt>. Acesso em: 17 out. 2016.

SÁ, Guilherme José da Silva. "Meus macacos são vocês": um antropólogo seguindo primatólogos em campo. Revista ANTHROPOLÓGICAS, Recife, v. 16, p. 
41-66, 2005. Disponível em:

$<$ http://www.biolinguagem.com/ling cog cult/sa meusmacacos 2005.pdf > . Acesso em: 10 out. 2016.

SOUSA SANTOS, Boaventura. Um Discurso sobre as Ciências. Porto: Edições Afrontamento, 2002.

TOLEDO, Victor M.; BARRERA-BASSOLS, Narciso. A etnoecologia: uma ciência pós-normal que estuda as sabedorias tradicionais. In: SILVA, ValdelineAtanazio da; ALMEIDA, Alyson Luiz Santos; ALBUQUERQUE, Ulysses Paulino. Etnobiologia e Etnoecologia: pessoas \& natureza na América Latina. 1. ed. Recife: NUPEEA, 2010. p. 13-36.

VAN WASSENHOVEN, Michel. Evidências da eficácia da homeopatia. Cultura Homeopática, São Paulo, v. 20, p. 27-31, 2007. Disponível em:

http://www.feg.unesp.br/ ojs/index.php/ijhdr/article/viewFile/25/18. Acesso em: 27 out. 2016.

\section{Artigo:}

Recebido em 10 de Novembrode 2016.

Aceito em 06 de Julho de 2017. 